\title{
Ladder resonator: A novel superconducting structure for the very low $\beta$ part of high current linacs
}

\author{
V. Andreev, G. Bisoffi, A. Pisent, E. Bissiato, M. Comunian, and E. Fagotti \\ LNL-INFN, via Romea 4, I35020 Legnaro (PD), Italy \\ T. Shirai \\ ICR Kyoto University, Uji Kyoto 611, Japan \\ (Received 17 July 2002; published 22 April 2003)
}

\begin{abstract}
A novel superconducting (sc) structure is proposed, to accelerate a high intensity proton beam within the energy range of 5-20 MeV. The comparison with different sc structures from the point of view of both rf efficiency and technological aspects of manufacture looks promising. Results of M.A.F.I.A. simulations are given.
\end{abstract}

DOI: 10.1103/PhysRevSTAB.6.040101

PACS numbers: 29.17.+w, 29.27.-a, 41.75.-i

\section{INTRODUCTION}

High current proton linacs of the intensity range from 1 to $50 \mathrm{~mA}$ and the final energy from $100 \mathrm{MeV}$ to $2 \mathrm{GeV}$ are necessary for a variety of research and technology applications, namely, transmutation of nuclear wastes, production of radioactive ion beams, spallation neutron sources, neutrino factories, and technological neutron irradiation tools [1]. The basic linac architecture, and several technological developments related to it, can be shared by many of these applications.

The specific application considered in this paper is a proton driver for the production of exotic nuclear species, in the framework of the EURISOL design study (funded by the European Commission [2]) for a next generation ISOL facility in Europe.

The proton driver proposed for EURISOL is a $5 \mathrm{~mA} \mathrm{cw}$ linac composed by a normal conducting radio frequency quadrupole (RFQ), operating at $352 \mathrm{MHz}$, as injector, and by elliptical superconducting cavities, resonating at $704 \mathrm{MHz}$, in the higher energy linac section (85 MeV$1 \mathrm{GeV})$.

In the intermediate energy section $(5-85 \mathrm{MeV})$ an ISCL (independently phased superconducting cavity linac) is proposed, consisting of a few families of independently phased resonators operating at $352 \mathrm{MHz}$. This linac has investment costs comparable to the alternative room temperature solution [a drift tube linac (DTL)], but offers a factor 6 smaller ac power costs, smaller beam rms dimension to the beam port aperture (beneficial for safety considerations), and independent control of the resonator phase. The latter feature would allow acceleration of ions with a charge-to-mass ratio $q / \mathrm{A}=1 / 2$ or $1 / 3$, depending on the chosen geometry of the superconducting (sc) cavity. More in detail, the part beyond $15-20 \mathrm{MeV}$ can be conveniently implemented by using 2-gap HWR (half wave resonator), QWR (quarter wave resonator), or spoke resonators, while for the first part (5-20 MeV) a solid architecture is still to be demonstrated. DTL structure or linac based on sc reentrant cavities are possible solutions, having the disadvantages of high power dissipation and a large number of cavities and rf systems, respectively.

In this paper we propose the use of few sc 4-gap resonators of a novel kind for the energy range 5-20 MeV.

The sc cavities typically proposed for the $\beta$ range $0.1-$ 0.5 [3-9] are of the crossed-stem type. The current density over the cavity surface is quite evenly distributed in crossed-stem resonators, but there is no place in the cavity to put a large size flange at $B_{s, p}<50 \mathrm{G}$, considered a reasonable threshold to avoid excessive power dissipation at flanged joints in sc cavities. All the cavity niobium components must be eventually welded together: this seriously limits the access to the cavity internal volume, making inspections more troublesome and surface processes, such as chemical etching and high pressure water rinsing (HPWR), possible only through either the beam or the rf-coupling ports.

Moreover, in order to achieve flatness of the accelerating field along the gaps, 3-gap or multigap resonators of the crossed-stem type require extra space for the magnetic field in the first and last gap: this typically takes the total longitudinal space of one rf cell. This is particularly penalizing for the very low $\beta$ branch of the linac $(\beta=$ $0.1-0.2$ ), where three gaps at most can be accommodated in the limited focusing period available. Accelerating cavities with a maximum of one or two gaps have been proposed for the very low $\beta$ range so far [10-12].

In this paper, we propose the novel design of a $352 \mathrm{MHz}$ 4-gap sc resonator, which we have called a "ladder resonator." Such a cavity is capable of providing efficient acceleration in this velocity range, thus allowing one to significantly diminish the number of accelerating cavities and the overall linac length. The accelerating field is fully flat along the four gaps, leaving negligible extra space in the longitudinal direction.

The most significant and original feature in the cavity design is the possibility to equip the resonator with two 
large flanges, allowing easy internal inspections, surface treatments, and possible repairs.

\section{LINAC DESIGN}

The ladder resonator is proposed for the first section of a high current proton linac, following the $5 \mathrm{MeV}$ RFQ. The acceleration up to $20 \mathrm{MeV}$ is performed by two families of 4-gap cavities, with optimum $\beta_{0}=0.12$ and 0.17. Twelve cavities with an accelerating field of $5.8 \mathrm{MV} / \mathrm{m}$ are needed in total. The average synchronous phase is $-30^{\circ}$ and the effective length is $2 \beta_{0} \lambda$. The transit time factor (TTF) is always above $80 \%$ in this scenario (see Fig. 1).

The preliminary linac structure presented below uses a focusing-drift-defocusing-drift (FODO) with superconducting quadrupoles installed inside the cryomodules. The use of quadrupoles is homogeneous to what happens before (RFQ) and afterwards (superconducting linac with external quadrupole doublets), with a separate regulation of vertical and horizontal focusing directions. Moreover, compared to quadrupole doublet or solenoid focusing, the FODO solution has the advantage of an easier matching with the RFQ.

On the other hand the choice of this new cavity is not strictly linked to the choice of the focusing structure and the ladder can be used with similar advantages in a solenoid or doublet lattice.

The cavities are located in two different cryostats, one for each family, with a warm section, housing beam diagnostics, in between (Fig. 2). The transverse focusing is achieved using superconducting quadrupoles, with iron poles and superconducting windings, housed in the same cryostat. A prototype of such quadrupoles has been built at the Michigan State University according to LNL specifications for the TRASCO linac [13]. The use of quadrupole magnets, relatively close to high gradient superconducting cavities, is delicate, since the residual

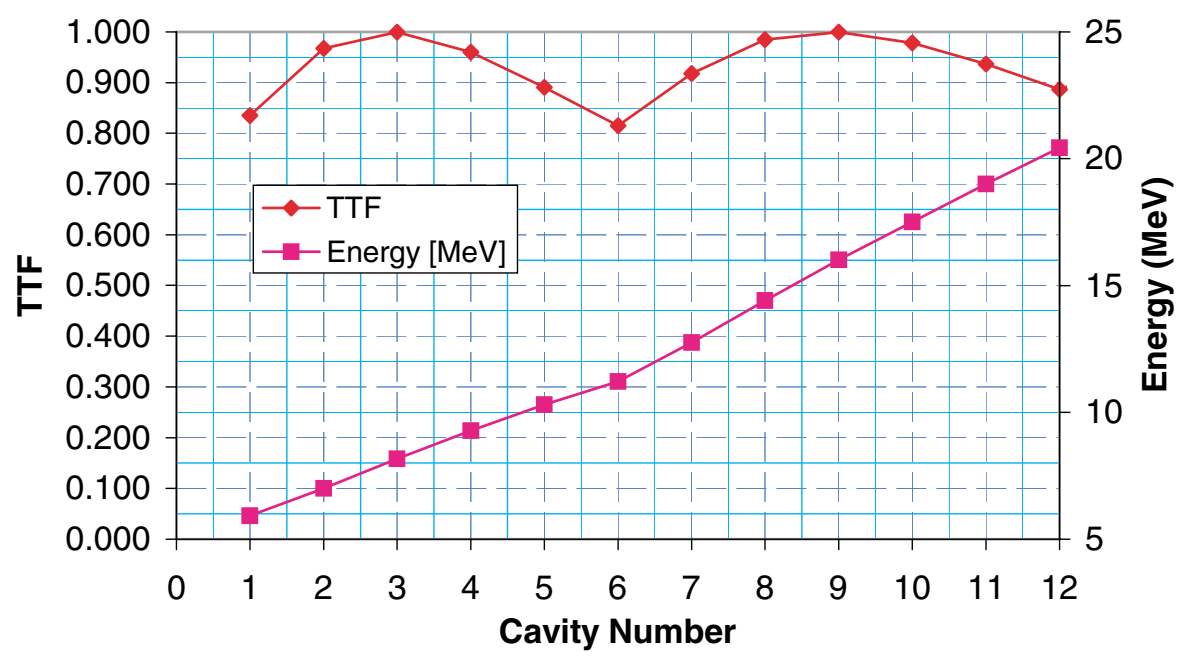

FIG. 1. (Color) Synchronous particle energy and transit time factor along the linac; the TTF is calculated for four ideal gaps, with optimum $\beta_{0}=0.12$ and 0.17 .

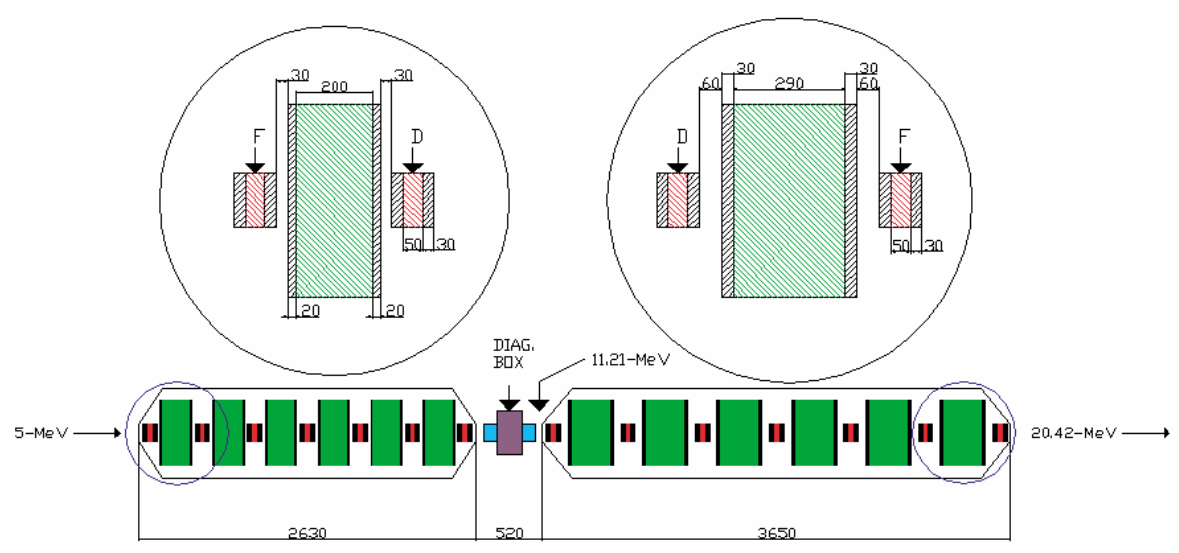

FIG. 2. (Color) Schematic layout of the ISCL (quotes are in $\mathrm{mm}$ ). The two cryostats house six ladder resonators (in green) of the two families, with sc quadrupoles (in red) in between. 


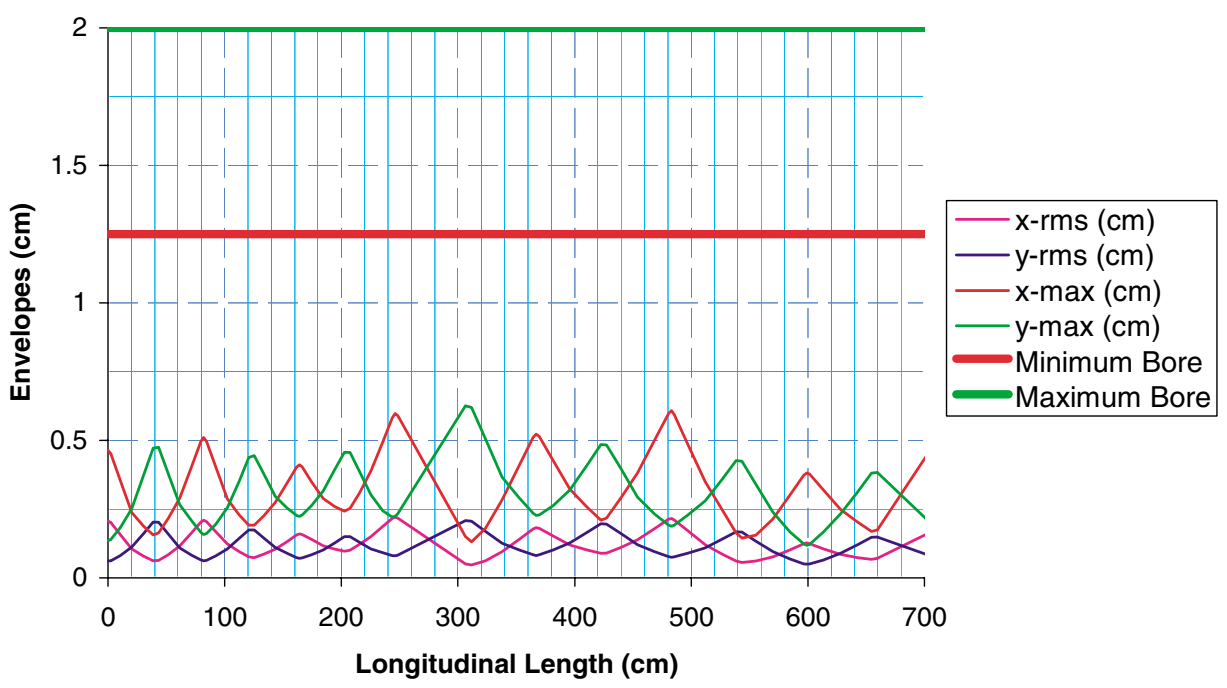

FIG. 3. (Color) Transverse beam envelopes (rms and maximum values) for $5 \mathrm{~mA}$ compared with maximum and minimum bore values.

magnetic field could be trapped by the cavity during start up. Indeed in our design, the calculated magnetic field on cavity even with the quadrupole at maximum field is below $0.5 \mathrm{G}$ (in the first cryostat that is more compact). This should be a safe value, and an experimental verification will be done at LNL.

The beam bore diameter for the quadrupoles is $40 \mathrm{~mm}$, for the cavities $25 \mathrm{~mm}$. The focusing structure has a constant period length in each cryostat, but with a longer period in the second cryostat where the cavities themselves are longer. Corresponding to the warm transition there is a long period without any rf defocusing.

The beam matching has to cope with these changes, and with space charge effect and possible single particle and envelope resonances. In particular, the parametric resonance, occurring when the longitudinal phase advance is about twice the transverse phase advance, must be avoided to preserve beam quality. Parametric resonance has a fast exponential growth that rapidly spoils the transverse emittance; to avoid this effect a strong transverse focusing has been implemented in the first cells (about $97^{\circ}$ per FODO period).

A rough estimate of the resonance width gives the prescription $0.4 \leq\left(\sigma_{T} / \sigma_{L}\right) \leq 0.6$. The longitudinal phase advance per lattice period $L$ is

$$
\begin{aligned}
\sigma_{L} & =L \sqrt{\frac{e E}{m c^{2}} \frac{2 \pi \sin \left(-\phi_{s}\right)}{\beta^{3} \gamma^{3} \lambda}} \approx L \sqrt{\frac{e n \Delta W}{m c^{2} L} \frac{2 \pi \sin \left(-\phi_{s}\right)}{\beta^{3} \gamma^{3} \lambda}} \\
& \propto \sqrt{L},
\end{aligned}
$$

with $E$ the average accelerating field, $n$ the number of cavities per period, $\beta$ and $\gamma$ relativistic parameters, and $\lambda$ the $\mathrm{rf}$ wavelength. The longitudinal phase advance is larger at lower energy and is proportional to $\sqrt{L}$. The transverse phase advance per period $\sigma_{T}$ is limited to $\pi / 2$ due to envelope instability. As a consequence $\sigma_{L} \leq$ $\left(\sigma_{T} / 0.4\right) \leq(\pi / 0.8)$ determines a limit on the possible period length $L$ (for a given number of cavities and energy gain per cavity). In other words, the use of high performance cavities (high $\Delta W$ ), that is economically advantageous, requires the compactness of the period and of the cavities themselves.

Beam envelopes corresponding to a $5 \mathrm{~mA}$ current are plotted in Fig. 3. The simulation is performed with PARMILA [14] using 100000 macroparticles. Cavities are simulated with rf gaps; the initial rms emittances, corresponding to the output of the TRASCO RFQ, are $0.2 \mathrm{~mm}$ mrad normalized for the transverse planes, and $0.2 \mathrm{MeV}$ deg for the longitudinal plane. The space charge effect is not dominant (initial transverse tune depression is about 10\%) and the same beam matching gives full transmission for a large range of beam current (at least up to $50 \mathrm{~mA}$ ).

This opens a wide range of future applications and suggests that for a $5 \mathrm{~mA}$ beam current (EURISOL and SPES projects), the limitation in the current is due to the available rf power and not to beam dynamics.

To guarantee the operation of the linac with losses below $1 \mathrm{~W} / \mathrm{m}$, a value which is needed for hands-on maintenance, deeper studies are needed. The use of a larger number of particles, a better modeling of the $\mathrm{rf}$ field, a systematic study of alignment errors, and analysis with a fully 3D space charge routine are all elements needed for the final simulations.

Preliminary results for a $90 \mathrm{MeV}$ linac, based on this low energy part and followed by eight cryostats with 2-gap resonators, has been presented in Refs. $[15,16]$. In particular, simulations done with HALODYN code (3D Poisson solver), with $20 \times 10^{6}$ particles and $5 \mathrm{~mA}$, show full transmission and no emittance growth up to the final energy. 


\section{CAVITY DESIGN REQUIREMENTS}

Some preliminary considerations on the rf parameters and construction issues are necessary for the optimization of the 4-gap resonator geometry.

First of all, it is reasonable to assume not to exceed a maximum surface electric field of $E_{s, p}=30 \mathrm{MV} / \mathrm{m}$ in the design. This reflects both our expertise with the tests of a sc RFQ [17] [a more complicated structure than the one proposed here with a number of electron-beam (e-b) welds exceeding 100], and the measured $Q$ curve of the very first 2-gap sc spoke cavity $(\beta=0.2)$ (designed and produced at Argonne [18], tested in April 2001 at Los Alamos [7]).

As for the maximum surface magnetic field $B_{s, p}$, we decided not to exceed $0.065 \mathrm{~T}$, which is commonly accepted as a still reasonable value $[6,11,19]$.

In optimizing a sc 4-gap resonator and in comparing different design options, it is sensible to be limited by whatever of either the electric or the magnetic field limits is reached first.

Aiming at compactness (or acceleration efficiency), the accelerating field is a fundamental figure of merit in the design. Consequently, the best possible flatness in the accelerating field along the four gaps is a crucial figure of merit of the resonator.

In consideration of the novelty of the structure and the large number of e-b welds, it is desirable to ensure easy access to the interior volume after the construction is complete. This would allow easy inspection and treatment of surfaces exposed to the rf fields, including possible mechanical repair, chemical etching, or HPWR. This is possible only if flanged joints, in a region of low enough current density (where $B_{s}<50 \mathrm{G}$ [20]), can be included in the design.

Last but not least, the frequency separation of the working mode from the higher order modes must remain large enough to keep the voltage distribution over the accelerating gaps flat enough, taking into account construction errors.

\section{DESCRIPTION OF THE LADDER RESONATOR GEOMETRY}

We propose the 4-gap structure (shown in the cutaway view of Fig. 4 and in the 2D views of Fig. 5) as a good compromise among the above-mentioned requirements. Figure 5 shows two bidimensional cross sections of the same resonator. The resonator geometric parameters are shown in Table I.

The cavity tank is $450 \mathrm{~mm}$ in both height and width and has a $200 \mathrm{~mm}$ internal length. The stems are disposed in a ladder-type pattern. They are smaller in the center, where a $25 \mathrm{~mm}$ beam bore is made in the flat part of the cross section, and larger at the extremes, thus allowing a current distribution over a wider area. This reduces the magnetic surface field at the stem base, where

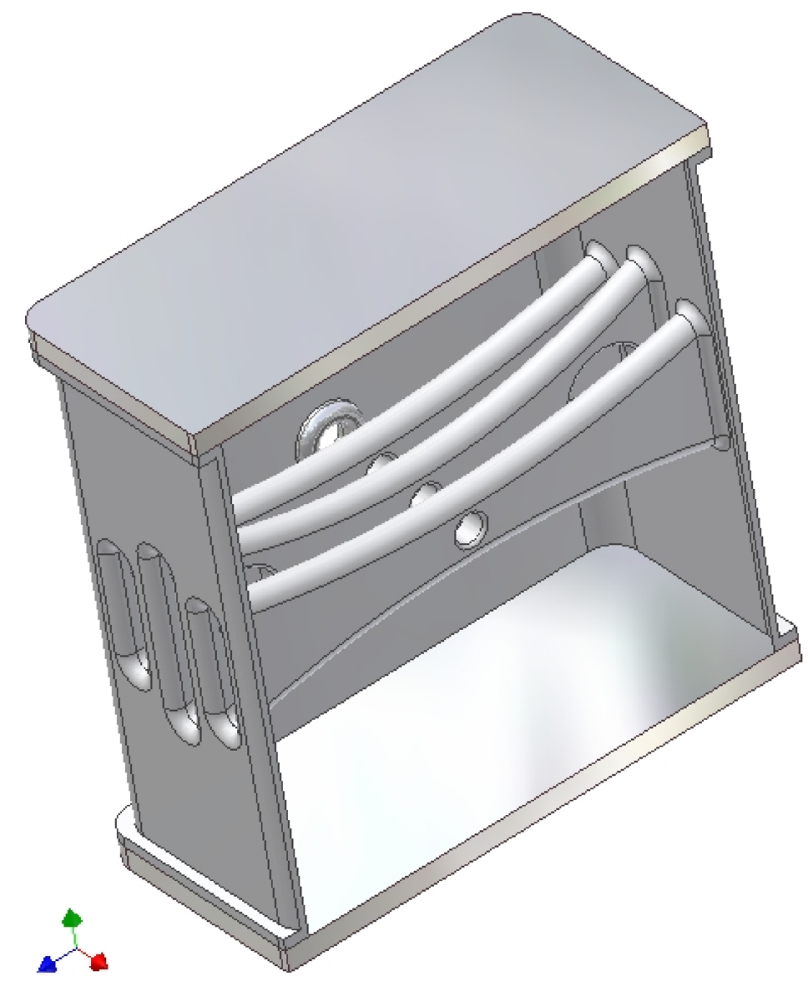

FIG. 4. (Color) Cutaway of the proposed ladder resonator and two cross sections. Different tapering of the central stem with respect to the other two and $11 \mathrm{~mm}$-long beam ports give full voltage flatness over the four gaps. Two coupling holes, close to the basis of the central stems, ensure a $4 \mathrm{MHz}$ splitting between the operational $\pi$ mode and the next higher mode.

it is higher. The enlargement angle is $8^{\circ}$ for the first and last stems and $14^{\circ}$ for the central one: this difference is sufficient to allow flatness of the accelerating fields, with minimal elongation (11 $\mathrm{mm}$ only) of the first and last cells with respect to the central ones.

Degeneration of the accelerating $\pi$ mode and higher order modes is avoided, by making two coupling holes on the central stem $(70 \mathrm{~mm}$ in diameter) in symmetric positions with respect to the beam axis.

TABLE I. Ladder resonator geometric parameters $\left(\beta_{0}=0.12\right)$.

Inner length along beam axis

Stem length

Internal height

Beam bore diameter

Gap length

Stem thickness

Stem width at aperture

Stem width at base (central stem)

Stem width at base (1st and 3rd stems)

rf coupling holes diameter
$200 \mathrm{~mm}$

$450 \mathrm{~mm}$

$450 \mathrm{~mm}$

$25 \mathrm{~mm}$

$25 \mathrm{~mm}$

$26 \mathrm{~mm}$

$65 \mathrm{~mm}$

$166 \mathrm{~mm}$

$125 \mathrm{~mm}$

$70 \mathrm{~mm}$ 

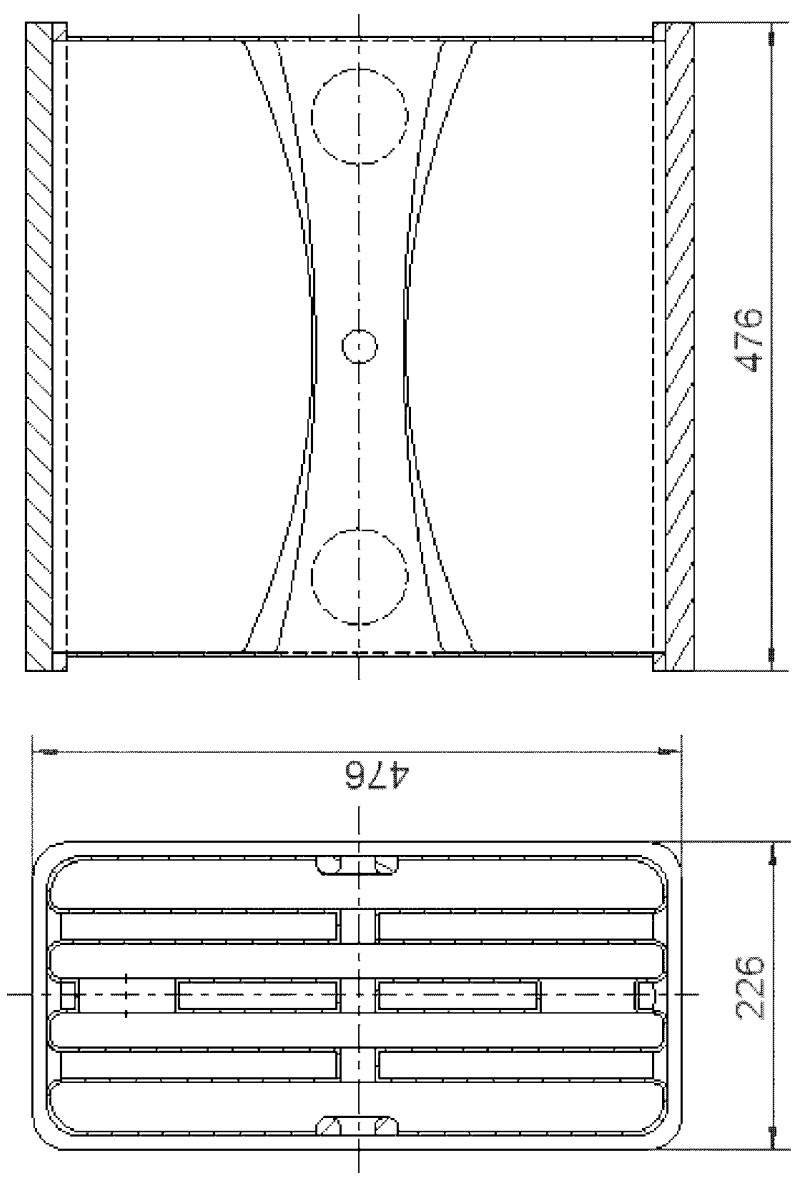

FIG. 5. 2D cross section and front view of the ladder cavity; a preliminary estimation of the external size is shown in the plot.

The large lateral ports are located at $225 \mathrm{~mm}$ from the beam axis: on their perimeter $B_{s}$ is sufficiently small to make the losses at the flanged joints negligible. The flanges allow easy access for inspection and surface treatments into the inner volume: they are flat and can be made in copper, covered by a thin sc layer of sputtered niobium.

The resonator stems and tank can be built in full $\mathrm{Nb}$. The stems could be first machined in two parts by drop hammer forming and then joined together by two oppo- site longitudinal e-b welds. Afterwards drift tubes and coupling holes can be easily machined. Technical solutions exist, to e-b weld the stems to the tank from either the inside or the outside; $2-\mathrm{mm}$ thick end plates, previously equipped with (e-b) welded beam ports, can be pushed or pulled for fine frequency tuning. Curvature radii of around $8 \mathrm{~mm}$ should be kept, both at the stem bases and at the sides of the end plates facing them, since in those locations current density is fairly high.

\section{M.A.F.I.A. RESULTS}

The shape of the ladder resonator shown in Fig. 4 has been optimized with the M.A.F.I.A. code [17]. The optimization aimed at (i) achieving full voltage flatness; (ii) finding the appropriate place to put flanged joints; (iii) staying within the peak surface field limits discussed in paragraph 3, while ensuring sufficient total accelerating voltage; (iv) designing a cavity easy to build; (v) and ensuring sufficiently large mode separation.

The main results of the simulations are summarized in Table II.

As can be seen in Fig. 6, the peak surface field of $0.065 \mathrm{~T}$ is reached at the coupling holes: we set this as the limiting value, to which all other electromagnetic values are normalized. A smaller hole diameter would give a smaller $B_{s, p}$, but also lower rf coupling. A $100 \%$ flatness of the accelerating field over the four gaps is achieved, as shown in Fig. 7. The TTF, as a function of $\beta$, is plotted in Fig. 8. Such a function can be approximated with a cubic polynomial:

$$
\operatorname{TTF}(\beta)=-11.95+268.01 \beta-1829.8 \beta^{2}+4026.9 \beta^{3},
$$

with $10^{-4}$ precision, or with the formula relative to four thin gaps (with an effective $\beta_{0}=0.123$ ):

$$
\operatorname{TTF}(\beta)=\sin \left(\frac{\pi}{2} \frac{0.123}{\beta}\right)-\sin \left(\frac{3 \pi}{2} \frac{0.123}{\beta}\right)
$$

with $10^{-2}$ precision.

TABLE II. Ladder resonator rf parameters.

\begin{tabular}{cc}
\hline \hline$B_{s, p}$ & $0.065 \mathrm{~T}$ (set as limit) \\
$E_{s, p} \beta_{0}=0.12$ & $20 \mathrm{MV} / \mathrm{m}$ \\
Energy gain at $\beta^{2}$ & $1.15 \mathrm{MeV} / q$ \\
Accelerating field $E_{0}$ & $5.8 \mathrm{MV} / \mathrm{m}$ \\
$U / E_{a}^{2}$ & $0.059 \mathrm{~J} /(\mathrm{MV} / \mathrm{m})^{2}$ \\
$\mathrm{rf}$ coupling & $1.2 \%$ \\
$Q-\mathrm{K}$ (assumed) & $5 \times 10^{8}$ \\
$\Gamma$ & $44.75 \Omega$ \\
$B_{s, p} / E_{0} \mathrm{~T}$ & $0.0112 \mathrm{~T} / \mathrm{MV} / \mathrm{m}$ \\
$\mathrm{rf}$ power dissipation $\left(@=5 \times 10^{8}\right)$ & $10 \mathrm{~W}$ \\
$B$ at the flanged joint & $0.0015 \mathrm{~T}$ \\
Accelerating field flatness (between central and end cells) & $100 \%$ \\
\hline \hline
\end{tabular}




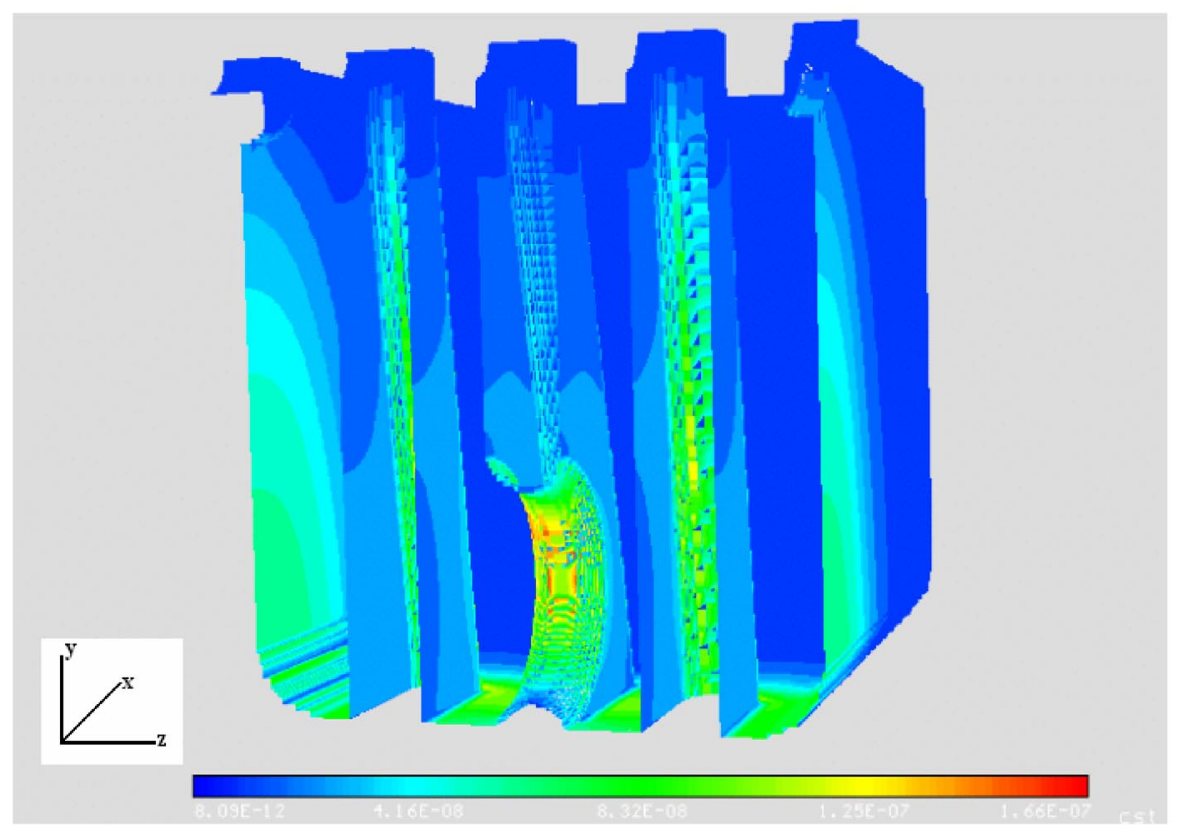

FIG. 6. (Color) 3D plot of the magnetic field on a quarter of the resonator internal surface, as calculated by M.A.F.I.A. It can be noted that the higher fields are concentrated in the portion of the resonator among the stems and they are vanishing towards the $x$ direction, where it was chosen to locate the flanged joint; $B_{s, p}$ is located in the coupling hole.

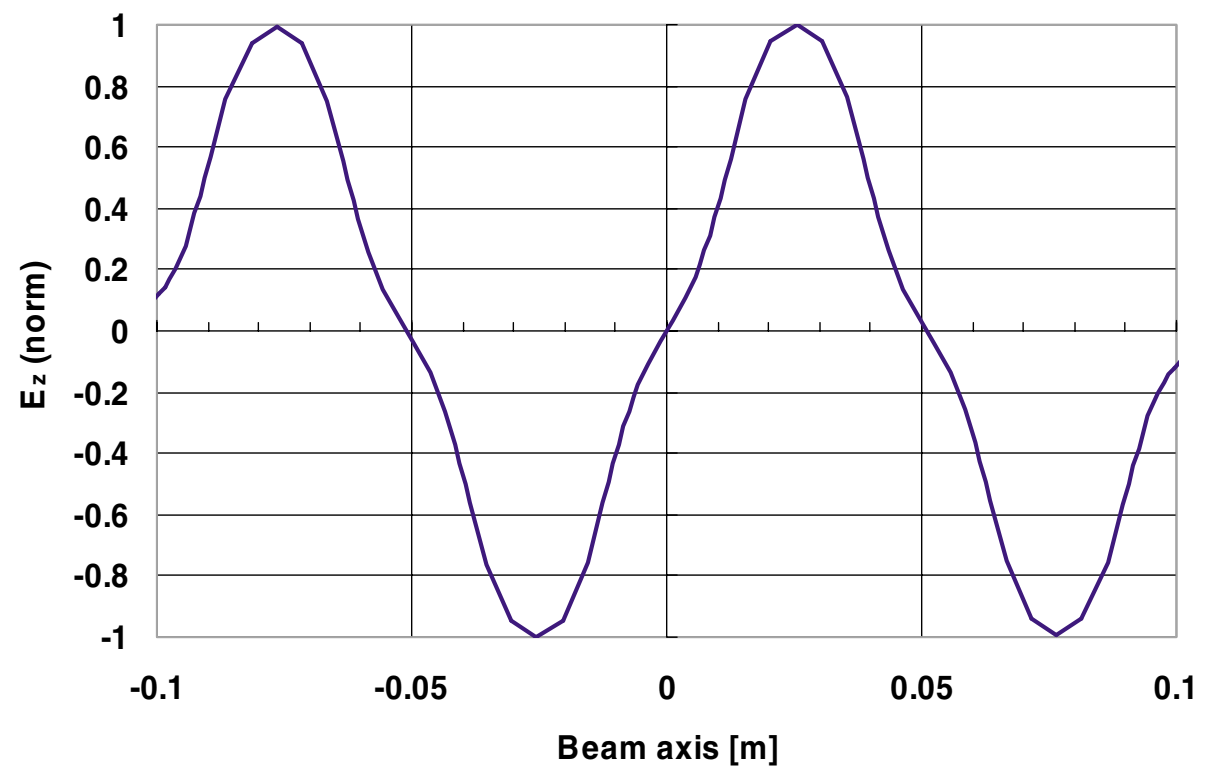

FIG. 7. (Color) Distribution of $E_{z}$ along the beam axis, showing that full field flatness is achieved.

A $4 \mathrm{MHz}$ mode separation (i.e., a $1.2 \%$ coupling), allowed by these coupling holes, is sufficient to allow a reliable construction with realistic specifications. Figure 9 shows a schematic representation of the four lowest modes of the ladder resonator. As it has been checked via simulations, with the proposed design a positioning error of $0.1 \mathrm{~mm}$ of one of the stems in the longitudinal direction causes a deviation of about $1 \%$ in the flatness of the accelerating field, accompanied by an increase of the mode separation by $1.5 \%$. While the flatness deviation is more than acceptable, experience, made at INFN-LNL with the construction of sc RFQ's [21,22], shows that the specification of $0.1 \mathrm{~mm}$ in positioning can even be exceeded.

As shown in Table II, the accelerating field of the ladder cavity is limited by the peak surface magnetic field $B_{s, p}$ rather than by the peak surface electric field $E_{s, p}$. At the limit of $B_{s, p}=0.065 \mathrm{~T}, E_{s, p}$ reaches $20 \mathrm{MV} / \mathrm{m}$ : a total 


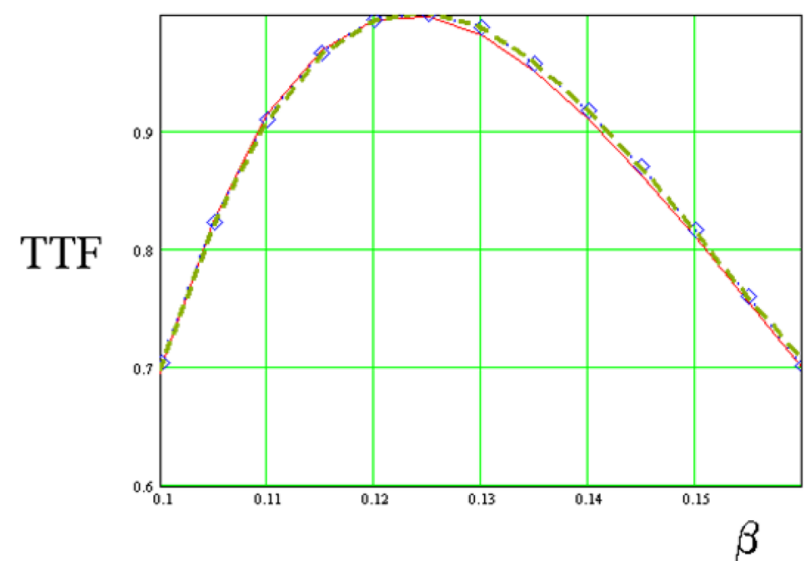

FIG. 8. (Color) TTF curve of the ladder resonator (blue diamonds), plotted together with the polynomial approximation [Eq. (1), green dashed curve] and the 4-thin-gap approximation [Eq. (2), red solid curve].

accelerating voltage of $1.15 \mathrm{MV}$ is hence achievable, which corresponds to an accelerating field $E_{a}=$ $5.8 \mathrm{MV} / \mathrm{m}$. This value is comparable to experimental or design values so far reported for resonators of the entire $\beta=0.1-0.5$ range [4-7,9].

Moreover, while the latter are resonators with only one or two gaps, the ladder cavity is the only 4-gap structure which is enough compact to be proposed from the very lowest velocity range $(\beta=0.1-0.2)$, i.e., immediately following the RFQ.

As a consequence the real estate gradient in this region is significantly larger using ladder resonators with respect to alternative geometries, which has a clear benefit on linac compactness and construction costs.

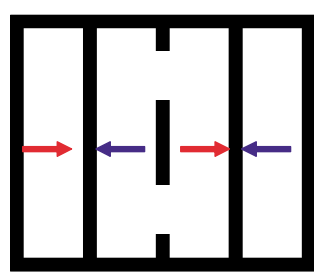

$\mathrm{f}_{0}=352 \mathrm{MHz}$ (working mode)

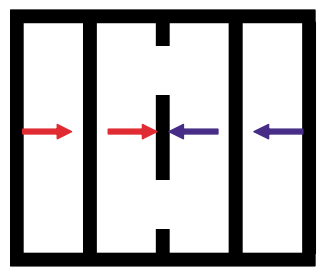

$\mathrm{f}_{2}=357.2 \mathrm{MHz}$

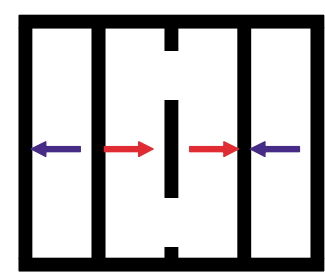

$\mathrm{f}_{1}=356.3 \mathrm{MHz}$

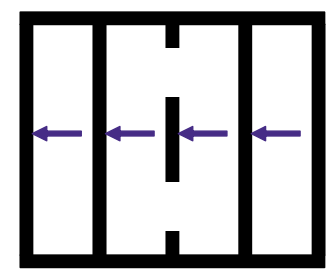

$\mathrm{f}_{3}=449.1 \mathrm{MHz}$
FIG. 9. (Color) Schematic representation of the first four frequency modes of the ladder structure.

\section{CONCLUSIONS}

We propose the ladder resonator as a very competitive structure for the very low energy section of a high current proton linac $(\beta>0.1)$, where high acceleration compactness is a fundamental issue.

In the calculated available space of $0.2 \mathrm{~m}$ (resonator internal length), it is possible to design a ladder resonator with four gaps. Since the inductance of the first and last cells of ladder resonators is very close to that of the central ones, it is possible to allocate four gaps, in that limited space, with $100 \%$ flatness of the accelerating field.

Mode degeneration of the ladder resonator is eliminated by the introduction of two coupling holes in the central stem. This is the location where the magnetic field is the maximum.

Taking $E_{s, p}=30 \mathrm{MV} / \mathrm{m}$ and $B_{s, p}=0.065 \mathrm{~T}$ as design limit values, the 4-gap ladder resonator achieves a $5.8 \mathrm{MV} / \mathrm{m}$ accelerating field, where $B_{s, p}$ is the limit.

Since the current density distribution on the tank of ladder cavities quickly drops, when moving away from the parallel stems, it is possible to introduce large flanged ports on both sides of the resonator, without causing any relevant electromagnetic losses at the joints. We regard this option as of fundamental importance, for efficient chemical polishing, rinsing, or simple inspection of the resonator during its life cycle.

A preliminary application of the ladder resonator has been calculated for the EURISOL driver linac [20]. Six resonators of the first $\beta$ family $\left(\beta_{\mathrm{opt}}=0.12\right)$ and five resonators of the second $\beta$ family $\left(\beta_{\text {opt }}=0.17\right)$ cover the energy range from 5 to $20 \mathrm{MeV}$, with transit time factor values always exceeding 0.8. They are followed by 94 2-gap resonators, of the spoke, the HWR, or the QWR type.

The construction technology is rather standard. The required positioning precision of the welded stems is less challenging than what has already been achieved at INFN-LNL in the construction of two sc RFQ's.

The design study of the ladder cavity will continue with (i) electromagnetic and mechanical analysis of the end plates, to be used as slow tuners of the resonant frequency; (ii) studies of a possible stiffening system, associated with a computational optimization of the mechanical structure with respect to the vibration eigenmodes; (iii) and technical drawing of a resonator prototype and of the liquid helium Dewar housing it, for sc tests to be performed in an existing test cryostat at INFN-LNL.

[1] J. M. Lagniel, in Proceedings of the European Particle Accelerator Conference, 2000, Vienna (http:// accelconf.web.cern.ch/AccelConf/e00/PAPERS/ THP5B05.pdf), p. 945.

[2] http://www.ganil.fr/eurisol 
[3] J. R. Delayen, in Proceedings of the 10th Workshop on RF Superconductivity, Tsukuba, 2001 (Ref. [4]).

[4] F. L. Krawczyk, K.C. D. Chan, R. Garnett, R. Gentzlinger, R. P. LaFave, J. P. Kelley, D. L. Schrage, and T. Tajima, in Proceedings of the 10th Workshop on RF Superconductivity, Tsukuba, 2001 (http:// conference.kek.jp/SRF2001/)

[5] K.W. Shepard and M. Kedzie, in Proceedings of the 10th Workshop on RF Superconductivity, Tsukuba, 2001 (Ref. [4]).

[6] G. Olry, J. L. Biarrotte, H. Saugnac, and J. Lesrel, in Proceedings of the 10th Workshop on RF Superconductivity, Tsukuba, 2001 (Ref. [4]).

[7] T. Tajima, K. C. D. Chan, R. L. Edwards, R. C. Gentzlinger, W. B. Haynes, J. P. Kelley, F. L. Krawczyk, J. E. Ledford, M. A. Madrid, D. I. Montoya, D. L. Schrage, and A. H. Shapiro, in Proceedings of the 10th Workshop on RF Superconductivity, Tsukuba, 2001 (Ref. [4]).

[8] R. Eichhorn, U. Ratzinger, and C. Peschke, in Proceedings of the 10th Workshop on RF Superconductivity, Tsukuba, 2001 (Ref. [4]).

[9] E. Zaplatin, W. Braeutigam, and R. Stassen, in Proceedings of the 10th Workshop on RF Superconductivity, Tsukuba, 2001 (Ref. [4]).

[10] M. Comunian, A. Facco, and A. Pisent, in Proceedings of the 19th International Linac Conference, Chicago, 1998 (http://accelconf.web.cern.ch/AccelConf/198/PAPERS/ MO4010.PDF), p. 64.

[11] A. Facco, V. Zviagintsev, and M. Pasini, in Proceedings of the 20th International Linac Conference, Monterey, 2000 (http://accelconf.web.cern.ch/ AccelConf/100/papers/THD11.pdf), p. 929.

[12] J-L. Biarrotte, J-L. Coacolo, J. Lesrel, and G. Olry, in Proceedings of the 2002 EPAC, La Villette-Paris (http:// accelconf.web.cern.ch/AccelConf/e02/PAPERS/ THPLE038.pdf), p. 1010.
[13] A. F. Zeller, J.C. DeKamp, A. Facco, T. L. Grimm, J. Kim, and R. Zink, LNL Annual Report No. LNLINDN(REP)-182/2002, 2001, p. 254.

[14] G. P. Boicourt, Los Alamos National Laboratory Report No. LA-UR-88-1544, 1988.

[15] A. Pisent, G. Bisoffi, M. Comunian, A. Facco, and E. Fagotti, in Proceedings of the 2002 EPAC, La Villette-Paris (Ref. [12]), p. 939.

[16] A. Franchi, M. Comunian, A. Pisent, A. Bazzani, S. Rambaldi, and G. Turchetti, "A 3D Poisson-Vlasov Code to Simulate the Space Charge Effects in the High Intensity TRASCO Linac, " in Proceedings of the 21st International Linac Conference, 2002, Gyeongju, Korea (to be published).

[17] T. Weiland, Part. Accel. 56, 61 (1996).

[18] K.W. Shepard, M. Kedzie, J. R. Delayen, J. Mammosser, and C. Piller, in Proceedings of the PAC99, New York (http://accelconf.web.cern.ch/AccelConf/p99/AI/ AIFRAME.HTM), p. 955.

[19] A. Facco and V. Zviagintsev, in Proceedings of the 2001 Particle Accelerator Conference, Chicago (http:// accelconf.web.cern.ch/AccelConf/p01/PAPERS/ MPPH136.PDF), p. 1095.

[20] G. Bisoffi, A. M. Porcellato, V. Andreev, G. Bassato, G. P. Bezzon, S. Canella, F. Chiurlotto, A. Lombardi, E. Chiaveri, W. Singer, T. Shirai, and S. Y. Stark, in Proceedings of the 2002 EPAC, La Villette-Paris (Ref. [12]), p. 266.

[21] G. Bisoffi, V. Andreev, G. P. Bezzon, F. Chiurlotto, M. Comunian, A. Lombardi, V. Palmieri, A. Pisent, A. M. Porcellato, S.Y. Stark, T. Shirai, W. Singer, and E. Chiaveri, in Proceedings of the European Particle Accelerator Conference, 2000, Vienna (Ref. [1]), p. 324.

[22] G. Bisoffi, in Proceedings of the 10th Workshop on RF Superconductivity, Tsukuba, 2001 (Ref. [4]). 